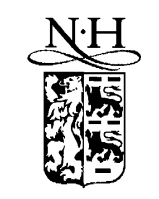

\title{
Non-linear behaviour of large-area avalanche photodiodes
}

\author{
L.M.P. Fernandes ${ }^{\mathrm{a}}$, J.A.M. Lopes ${ }^{\mathrm{a}}$, C.M.B. Monteiro ${ }^{\mathrm{a}}$, J.M.F. dos Santos ${ }^{\mathrm{a}}$, \\ R.E. Morgado ${ }^{\mathrm{b}, *}$ \\ a Physics Department, University of Coimbra, P-3004-516, Coimbra, Portugal \\ ${ }^{\mathrm{b}}$ Los Alamos National Laboratory, NM 87545, USA
}

\begin{abstract}
The characterisation of photodiodes used as photosensors requires a determination of the number of electron-hole pairs produced by scintillation light. One method involves comparing signals produced by X-ray absorptions occurring directly in the avalanche photodiode with the light signals. When the light is derived from light-emitting diodes in the 400-600 nm range, significant non-linear behaviour is reported. In the present work, we extend the study of the linear behaviour to large-area avalanche photodiodes, of Advanced Photonix, used as photosensors of the vacuum ultraviolet (VUV) scintillation light produced by argon $(128 \mathrm{~nm})$ and xenon $(173 \mathrm{~nm})$. We observed greater non-linearities in the avalanche photodiodes for the VUV scintillation light than reported previously for visible light, but considerably less than the non-linearities observed in other commercially available avalanche photodiodes. (C) 2002 Published by Elsevier Science B.V.
\end{abstract}

PACS: 29.40.M; 07.85.F; 85.60.D

Keywords: Scintillation detectors; X-ray detectors; Avalanche photodiodes

\section{Introduction}

Avalanche photodiodes (APDs) have assumed increasingly important instrumentation roles in broad areas of physics where their high quantum efficiency and low noise characteristics compete favourably with photomultiplier tubes. These include large electromagnetic calorimeters for high-energy physics at CERN [1-3], inorganic and plastic scintillator $\gamma$-ray spectrometers for

\footnotetext{
*Corresponding author. Physics Department, University of Coimbra, P-3004-516, Coimbra, Portugal. Tel.: + 351-239410667; fax: + 351-2398-29158.

E-mail address: rmorgado@lanl.gov (R.E. Morgado).
}

nuclear physics [4], and PET scanners [5] for medicine.

These applications have stimulated efforts to characterise the performances of several types of APDs that are now commercially available. An important characterisation is the number of electron-hole pairs produced per unit of absorbed energy, the knowledge of which enables a quantitative analysis of the noise sources contributing to the observed energy resolution. The number of electron-hole pairs is often determined by a comparison of the relative pulse amplitudes produced in the APD by the direct absorption of $\mathrm{X}$-rays with that produced by the scintillation light from a detector. The comparison is valid provided strict linearity between the initial number of 
electron-hole pairs and the resulting pulse amplitude is assumed throughout the range of avalanche multiplication gains.

Non-linearities have been reported, however, at high current densities and attributed to the spacecharge effects, reduced localised electric fields, and heating in the avalanche regions $[1,3,6,7]$ of the APD structure. As a result, the efficacy of using Xrays to determine the number of charge carriers produced in light measurements has been compromised to some degree.

The light sources used in these studies were light-emitting diodes in the $400-600 \mathrm{~nm}$ range, excluding the deep and vacuum ultraviolet (VUV) regions. With the commercial availability of windowless VUV-sensitive APDs, their instrumentation as photosensors of the scintillation light from noble gases and liquids for PET, X-, and $\gamma$ ray spectroscopy is under investigation [8-10].

In this work, we present for the first time, the results of a study to determine the non-linearities in large-area $\mathrm{APD}^{1}$ responses to the argon and xenon scintillations at $\sim 128$ and $\sim 173 \mathrm{~nm}$, respectively, from gas proportional scintillation counters. We compared the signals due to X-ray interactions occurring directly in the APD with signals from the scintillation light produced by Xray absorptions in the gas medium of the counter. The non-linearity is determined by comparing the amplitudes as a function of the APD reverse bias voltage.

\section{Description}

\subsection{Argon scintillation measurements}

\subsubsection{Detector and operation}

The argon gas proportional scintillation counter (GPSC) instrumented with a large-area APD is schematically depicted in Fig. 1. Its operational characteristics were described in Ref. [9]. X-rays entering the entrance window are absorbed primarily in the drift region by the photoelectric effect in argon. The cloud of primary electrons drifts

\footnotetext{
${ }^{1}$ Advanced Photonix, Inc., 1240 Avenida Acaso, Camarillo, CA 93012, USA.
}

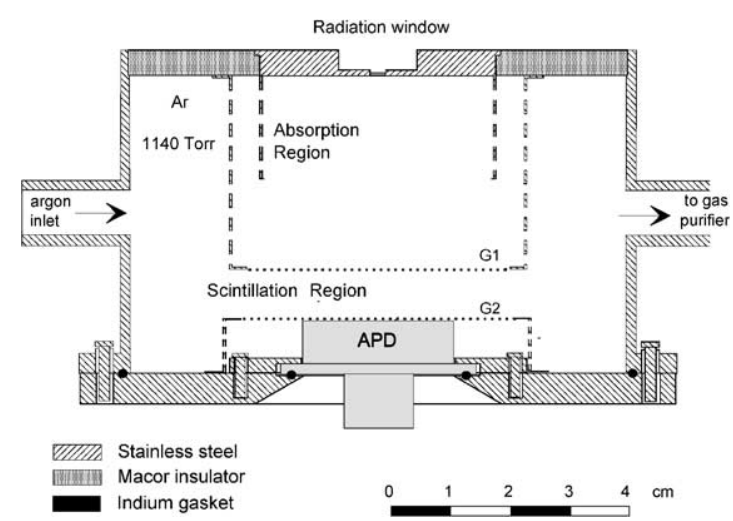

Fig. 1. The schematic of the GPSC with a large-area APD photosensor.

toward the scintillation region under the influence of the electric field established between the entrance window and grid G1. The reduced electric field in this region is chosen well below the scintillation and ionisation thresholds in argon.

In the scintillation region, the electrons are accelerated in the stronger electric field between G1 and G2, chosen above the scintillation threshold but below the ionisation threshold in argon. Each primary electron produces a number of VUV photons in the scintillation region. Proportionality between the incident $\mathrm{X}$-ray energy, the number of primary electrons, and the number of scintillation photons is maintained throughout the process.

The scintillation photons incident on the sensitive area of the APD produce electron-hole pairs in silicon that are multiplied by the avalanche process. The APD reverse bias voltage determines the multiplication gain in the avalanche process.

Concurrent with the acquisition of the detected signals from the absorption of X-rays in argon, a transmitted fraction of the X-rays are detected directly in the APD. The number of electron-hole pairs produced by direct absorption in the APD is determined from the energy of the X-ray and the $w$-value in silicon.

The total charge for each multiplication gain is integrated in a $1.5 \mathrm{~V} \mathrm{pC}^{-1}$ charge-sensitive preamplifier (Canberra 2006), followed by linear amplification (Hewlett Packard 5582A) with a 
2 - $\mu$ s shaping time. Pulse height analysis is performed with a 1024 channel multi-channel analyser (Nucleus PCA-II).

The pulse-height distribution is fit with a Gaussian function superimposed on a linear background. The pulse amplitude for each avalanche gain is determined from the centroid of the fitted Gaussian.

\subsubsection{Experimental results}

The measured pulse-height distribution for the $5.9 \mathrm{keV} \mathrm{X}$-rays from ${ }^{55} \mathrm{Fe}$ with the $\mathrm{K}_{\beta}$ line filtered by chromium is depicted in Fig. 2. Approximately $10 \%$ of the $5.9 \mathrm{keV} \mathrm{X}$-rays are transmitted through the intervening $3.3 \mathrm{~cm}$ of argon and $40 \%$ of these are absorbed in the depletion region of the APD.

The salient features of the pulse-height distribution include the $5.9 \mathrm{keV} \mathrm{X}$-ray peak from absorptions in the GPSC, the $5.9 \mathrm{keV}$ X-ray peak from direct absorptions in the APD, the escape peaks from the argon K-lines fluorescence, and the system electronic noise.

A direct comparison of the relative amplitudes from the argon scintillation and from the X-ray interaction in the APD can be made for each value of the APD gain.

In Fig. 3, the argon results are plotted as a ratio of the pulse amplitudes from X-ray interactions in

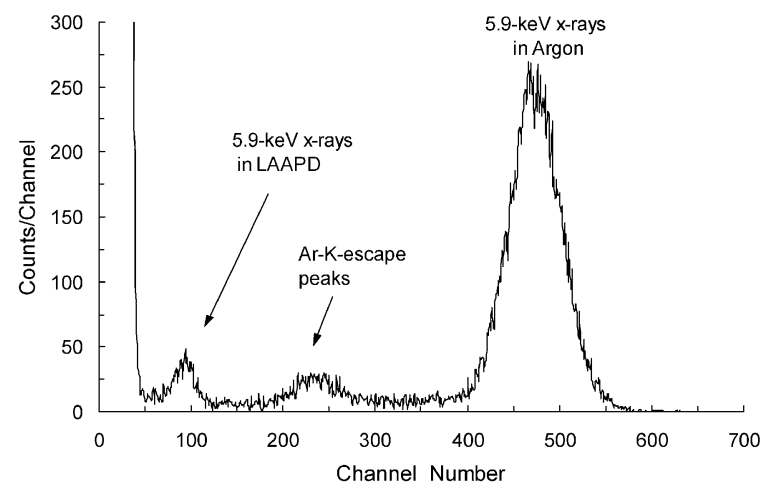

Fig. 2. The pulse-height distribution from an argon GPSC instrumented with a large-area APD for $5.9 \mathrm{keV}$ X-rays. $E / p$ of 0.2 and $5.0 \mathrm{~V} \mathrm{~cm}^{-1} \mathrm{Torr}^{-1}$ were used in the drift and scintillation region, respectively; the multiplication gain in the APD was approximately 40 .

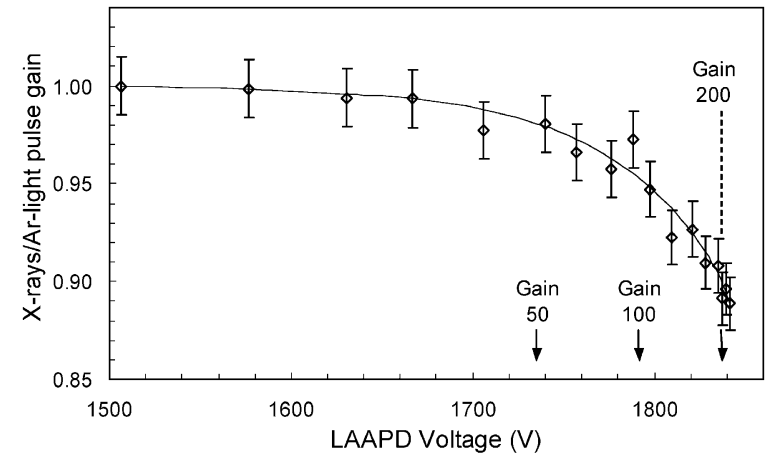

Fig. 3. The ratio of the pulse amplitudes in an argon GPSC instrumented with a large-area APD as a function of the APD reverse bias voltage.

the APD and in the GPSC, normalised to the maximum value, as a function of the APD reverse bias voltage. The deviation from linearity is greater than $10 \%$ for the APD gains above 200, 4 times larger than what was previously reported for visible light (around $600 \mathrm{~nm}$ ) [7]. For gains as low as 100 , deviations from linearity already exceed $4.5 \%$. Nevertheless, the non-linearity in the Advanced Photonix APD is still smaller than what has been observed with other types of APDs for visible light [7].

\subsection{Xenon scintillation measurements}

\subsubsection{Detector and operation}

The xenon GPSC instrumented with the largearea APD is schematically depicted in Fig. 4. The GPSC is a driftless design with a $1.1 \mathrm{~cm}$ deep

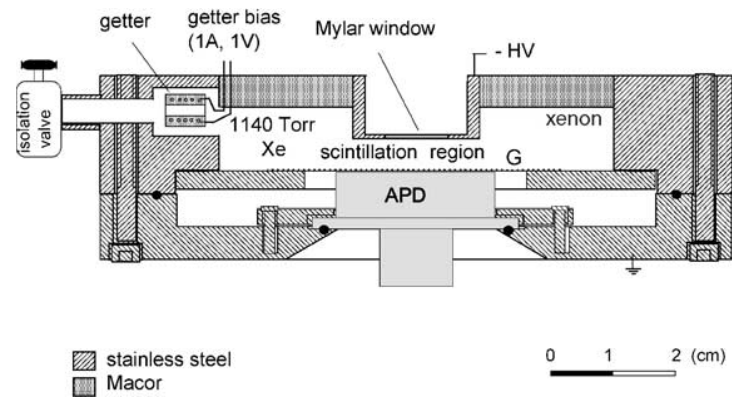

Fig. 4. A xenon driftless GPSC instrumented with a large-area APD. 
scintillation region. The APD is positioned just below the electron-collection grid $\mathrm{G}$. The entrance window is maintained at negative potential while grid $\mathrm{G}$ and the APD enclosure are maintained at ground potential.

The operation of the xenon driftless GPSC is similar to what has been previously described above with the notable exception that X-rays are now absorbed primarily in the scintillation region. The total number of scintillation photons produced by the primary electron cloud, however, will now depend on how deep into the scintillation region the $\mathrm{X}$-ray-absorption event occurs.

Although the driftless design results in degraded energy resolution for scintillation events, it allows higher transmission of the $5.9 \mathrm{keV} \mathrm{X-rays} \mathrm{through}$ the xenon and, therefore, more direct X-ray interactions in the APD. The charge collection and processing of the signals generated in xenon and directly in the APD are the same as already described above for argon.

\subsubsection{Experimental results}

Fig. 5 depicts the pulse-height distribution of $5.9 \mathrm{keV}$ X-rays from chromium-filtered ${ }^{55} \mathrm{Fe}$ measured in the xenon driftless GPSC. Approximately $0.2 \%$ of the $5.9 \mathrm{keV} \mathrm{X}$-rays are transmitted through the $1.1 \mathrm{~cm}$ of xenon, of which $40 \%$ are absorbed in the depletion region of the APD.

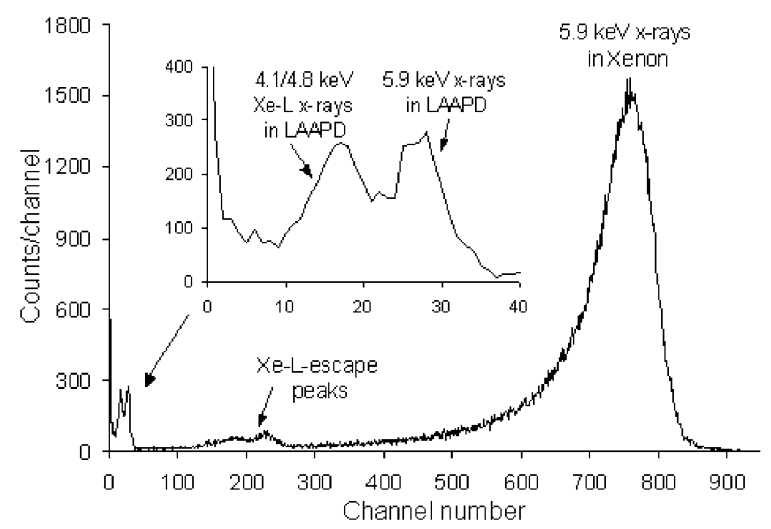

Fig. 5. Pulse-height distribution of $5.9 \mathrm{keV}$ X-rays from xenon driftless GPSC instrumented with a large-area APD. An $E / p$ of $5.5 \mathrm{~V} \mathrm{~cm}^{-1} \mathrm{Torr}^{-1}$ was used in the scintillation region; the multiplication gain in the APD was approximately 100 .
The salient features of the pulse-height distribution include the $5.9 \mathrm{keV}$ X-ray full-energy peak from absorptions in the xenon GPSC, the xenon $\mathrm{L}_{\alpha}$ and $\mathrm{L}_{\beta}$ escape peaks from $5.9 \mathrm{keV} \mathrm{X}$-ray absorptions in the xenon GPSC, the $5.9 \mathrm{keV} \mathrm{X}$ ray peak from absorptions in the APD, the 4.1 and $4.8 \mathrm{keV}$ xenon $\mathrm{L}_{\alpha}$ and $\mathrm{L}_{\beta}$ fluorescence peaks from absorptions in the APD, and the system electronic noise.

The distribution enabled a comparison between the pulse amplitudes resulting from the xenon scintillation interaction and the X-ray interactions in the APD as a function of the APD gain.

To extend the determination of non-linearity to higher energy X-rays, the pulse-height distributions for the $22.1 \mathrm{keV}$ X-rays from ${ }^{109} \mathrm{Cd}$ were also measured and analysed in the xenon driftless GPSC.

In Fig. 6, we plot the ratio of the pulse amplitudes from the X-ray interactions in the APD and from the GPSC, normalised to the maximum value, as a function of the APD reverse-bias voltage. For $5.9 \mathrm{keV} \mathrm{X}$-rays, the variation is only $\sim 6 \%$ for the APD gains of 200 and $\sim 3.5 \%$ for gains of about 100 . These values, though less than those measured for the argon scintillation, are a factor of 2 larger than for visible light (around $600 \mathrm{~nm}$ ) [7]. The results for $22.1 \mathrm{keV} \mathrm{X}$-rays demonstrate even greater nonlinearity.

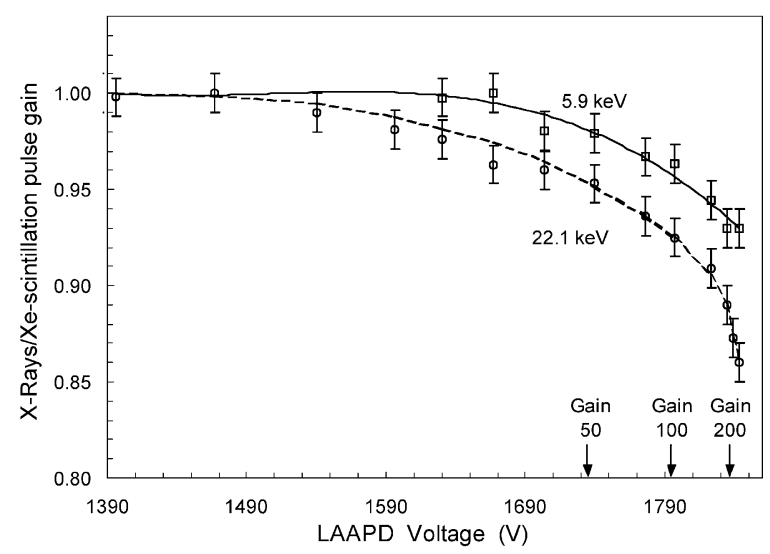

Fig. 6. The ratio of the pulse amplitudes in an xenon driftless GPSC instrumented with a large-area APD as a function of the APD reverse-bias voltage for 5.9 and $22.1 \mathrm{keV}$ X-rays. 


\section{Analysis and conclusions}

We have confirmed the non-linear behaviour of the Advanced Photonix large-area APDs for the VUV scintillations from argon and xenon. The results suggest a dependence of the APD linearity on wavelength as well: the scintillation light from argon $(128 \mathrm{~nm})$ demonstrated the largest nonlinearity of $10 \%$ at a gain of 200 , compared to $6 \%$ for xenon $(173 \mathrm{~nm})$, and $3 \%$ for visible light $(600 \mathrm{~nm})$ [7].

An accurate determination of the number of charge carriers produced in an APD by the VUV scintillation light may be compromised if strict linearity is assumed with direct X-ray interactions. Nevertheless, the non-linearity reported here for the VUV light in Advanced Photonix large-area APDs is less than what is observed in other types of APDs for visible light [7].

\section{Acknowledgements}

This work was carried out in the Atomic and Nuclear Instrumentation Group of the Instrumentation Center (Unit 217/94). R.E. Morgado acknowledges the support of the Calouste Gulbenkian Foundation for his professorship grant. Support is acknowledged from project CERN/ $\mathrm{P} / \mathrm{FIS} / 15200 / 99$.

\section{References}

[1] J.P. Pansart, Nucl. Instr. and Meth. A 387 (1997) 186.

[2] A. Karar, Y. Musienko, J.C. Vanal, Nucl. Instr. and Meth. A 428 (1999) 413.

[3] K. Deiters, et al., Nucl. Instr. and Meth. A 442 (2000) 193.

[4] R. Farrel, et al., Nucl. Instr. and Meth. A 387 (1997) 194.

[5] A. Ruru Chen, et al., Nucl. Instr. and Meth. A 433 (1999) 637.

[6] C.P. Allier, et al., IEEE Trans. Nucl. Sci. NS-45 (1998) 576.

[7] M. Moszynski, et al., Comparative study of avalanche photodiodes with different structures in scintillation detection, Presented at the IEEE Nuclear Science Symposium, Lyon, France, 15-20 October, 2000.

[8] J.A.M. Lopes, J.M.F. dos Santos, R.E. Morgado, C.A.N. Conde, Xenon gas proportional scintillation counter with a UV-sensitive, large area avalanche photodiode, Presented at the IEEE Nuclear Science Symposium, Lyon, France, 15-20 October, 2000; IEEE Trans. Nucl. Sci. 48 (2001), in press.

[9] C.M.B. Monteiro, J.A.M. Lopes, J.M.F. dos Santos, C.A.N. Conde, An argon gas proportional scintillation counter with a UV avalanche photodiode scintillation readout, Presented at the IEEE Nuclear Science Symposium, Lyon, France, 15-20 October, 2000; IEEE Trans Nucl. Sci. 48 (2001), in press.

[10] V.N. Solovov, V. Chepel, A. Hitachi, M.I. Lopes, R. Ferreira Marques, A.J.P.L. Policarpo, Detection of scintillation light of liquid xenon with a LAAPD, Presented at the IEEE Nuclear Science Symposium, Lyon, France, 15-20 October, 2000; IEEE Trans Nucl. Sci. Symp. Conf. Rec., paper \#521. 